\title{
Rani distribution and its application
}

\begin{abstract}
In the present paper, a new one parameter lifetime distribution named, "Rani Distribution' has been proposed for modeling lifetime data from engineering and biomedical sciences. Its various statistical and mathematical properties including its shapes for varying values of parameter, moments and moments based measures, hazard rate function, mean residual life function, stochastic ordering, deviations from the mean and the median, Bonferroni and Lorenz curves, order statistics, Renyi entropy measure and stress-strength reliability have been studied. Both the maximum likelihood estimation and the method of moments have been discussed for estimating the parameter of the proposed distribution. A simulation study has been carried out and results are presented. A numerical example has been presented to test the goodness of fit of the proposed distribution and it has been found that it gives much closer fit than almost all one parameter lifetime distributions introduced in statistical literature.
\end{abstract}

Keywords: lifetime distributions, statistical and mathematical properties, parameter estimation, goodness of fit
Volume 6 Issue I - 2017

\section{Rama Shanker}

Department of Statistics, Eritrea Institute of Technology, Asmara, Eritrea

Correspondence: Rama Shanker, Department of Statistics, Eritrea Institute of Technology, Asmara, Eritrea, Email shankerrama2009@gmail.com

Received: May 19, 2017| Published: May 29, 2017

\section{Introduction}

In the present world the modeling and analyzing lifetime data are essential in almost all applied sciences including medicine, engineering, insurance and finance, amongst others. The two classical one parameter lifetime distributions which are popular and are in use for modeling lifetime data from biomedical science and engineering are exponential and Lindley introduced by Lindley. ${ }^{1}$ Shanker, et al., ${ }^{2}$ have detailed comparative study on modeling of lifetime data from various fields of knowledge and observed that there are many lifetime data where these two distributions are not suitable due to their shapes, nature of hazard rate functions, and mean residual life functions, amongst others. In search for new one parameter lifetime distributions which gives better fit than exponential and Lindley distributions, recently Shanker has introduced several one parameter lifetime distributions in statistical literature namely Akash, ${ }^{3}$ Shanker, ${ }^{4}$ Aradhana, ${ }^{5}$ Sujatha, ${ }^{6}$ Amarendra, ${ }^{7}$ Devya, ${ }^{8}$ Rama $^{9}$ and Akshaya ${ }^{10}$ and showed that these distributions gives better fit than the classical exponential and Lindley distributions. The probability density function (pdf) and the corresponding cumulative distribution function (cdf) of Akash, ${ }^{3}$ Shanker, ${ }^{4}$ Aradhana, ${ }^{5}$ Sujatha, ${ }^{6}$ Amarendra, ${ }^{7}$ Devya,${ }^{8}$ Rama $^{9}$ and Lindley ${ }^{1}$ distributions are presented in Table (1). It has also been discussed by Shanker that although each of these lifetime distributions has advantages and disadvantages over one another due to its shapes, hazard rate functions and mean residual life functions, there are still many lifetime data where these distributions are not suitable for modeling lifetime data from theoretical or applied point of view. Therefore, an attempt has been made in this paper to obtain a new lifetime distribution which is flexible than these one parameter lifetime distributions for modeling lifetime data in reliability and in terms of its hazard rate shapes.

The new one parameter lifetime distribution is based on a twocomponent mixture of an exponential distribution having scale parameter $\theta$ and a gamma distribution having shape parameter 5 and scale parameter $\theta$ with their mixing proportion $\frac{\theta^{5}}{\theta^{5}+24}$.

The probability density function (p.d.f.) of a new one parameter lifetime distribution can be introduced as

$$
f(x ; \theta)=\frac{\theta^{5}}{\theta^{5}+24}\left(\theta+x^{4}\right) e^{-\theta x} ; x>0, \theta>0
$$

We would call this distribution, "Rani distribution". This distribution can be easily expressed as a mixture of exponential $(\theta)$ and gamma $(5, \theta)$ with mixing proportion $\frac{\theta^{5}}{\theta^{5}+24}$. We have

$$
f(x, \theta)=p g_{1}(x)+(1-p) g_{2}(x)
$$

where $p=\frac{\theta^{5}}{\theta^{5}+24}, g_{1}(x)=\theta e^{-\theta x}$, and $g_{2}(x)=\frac{\theta^{5} x^{4} e^{-\theta x}}{24}$.

The corresponding cumulative distribution function (c.d.f.) of (1.1) can easily be obtained as

$$
F(x, \theta)=1-\left[1+\frac{\theta x\left(\theta^{3} x^{3}+4 \theta^{2} x^{2}+12 \theta x+24\right)}{\theta^{5}+24}\right] e^{-\theta x} ; x>0, \theta>0
$$

The graphs of the p.d.f. and the c.d.f. of Rani distribution for varying values of the parameter $\theta$ are shown in Figures $1 \& 2$. The p.d.f. of Rani distribution is monotonically decreasing. 
Table I pdf and cdf of Akash, Shanker, Aradhana, Sujatha,Amarendra, Devya, Rama, Akshaya and lindley distributions for $x>0, \theta>0$

\section{Distributions}

pdf

Probability density functions and cumulative distribution functions

Akash

cdf

$F(x)=1-\left[1+\frac{\theta x(\theta x+2)}{\theta^{2}+2}\right] e^{-\theta x}$

pdf

Shanker

cdf

pdf

Aradhana

cdf

pdf

Sujatha

$c d f$

pdf

Amarendra

cdf

pdf

$$
f(x)=\frac{\theta^{2}}{\theta^{2}+1}(\theta+x) e^{-\theta x}
$$

$$
F(x)=1-\left[1+\frac{\theta x}{\theta^{2}+1}\right] e^{-\theta x}
$$$$
f(x)=\frac{\theta^{2}}{\theta^{2}+1}(\theta+x) e^{-\theta x}
$$

$F(x)=1-\left[1+\frac{\theta x(\theta x+2 \theta+2)}{\theta^{2}+2 \theta+2}\right] e^{-\theta x}$

$$
f(x)=\frac{\theta^{3}}{\theta^{2}+\theta+2}\left(1+x+x^{2}\right) e^{-\theta x}
$$

$$
F(x)=1-\left[1+\frac{\theta x(\theta x+\theta+2)}{\theta^{2}+\theta+2}\right] e^{-\theta x}
$$$$
f(x)=\frac{\theta^{4}}{\theta^{3}+\theta^{2}+2 \theta+6}\left(1+x+x^{2}+x^{3}\right) e^{-\theta x}
$$

$$
F(x)=1-\left[1+\frac{\theta^{3} x^{3}+\theta^{2}(\theta+3) x^{2}+\theta\left(\theta^{2}+2 \theta+6\right) x}{\theta^{3}+\theta^{2}+2 \theta+6}\right] e^{-\theta x}
$$

cdf

$$
f(x)=\frac{\theta^{5}}{\theta^{4}+\theta^{3}+2 \theta^{2}+6 \theta+24}\left(1+x+x^{2}+x^{3}+x^{4}\right) e^{-\theta x}
$$

Devya

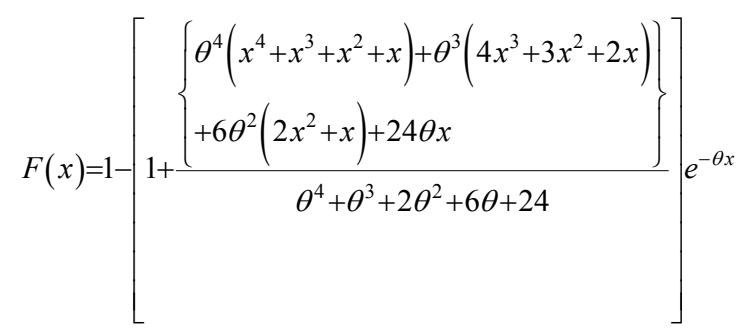

pdf

$$
f(x)=\frac{\theta^{4}}{\theta^{3}+6}\left(1+x^{3}\right) e^{-\theta x}
$$

Rama

cdf

$$
F(x)=1-\left[1+\frac{\theta^{3} x^{3}+3 \theta^{2} x^{2}+6 \theta x}{\theta^{3}+6}\right] e^{-\theta x}
$$


Table continued...

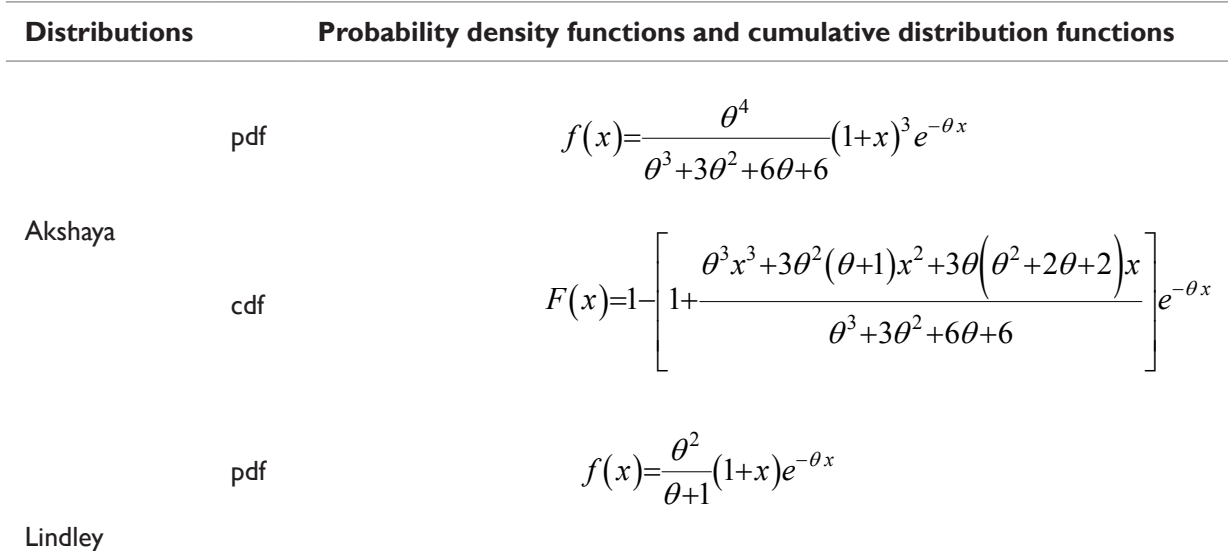

cdf

$$
F(x)=1-\left[1+\frac{\theta x}{\theta+1}\right] e^{-\theta x}
$$
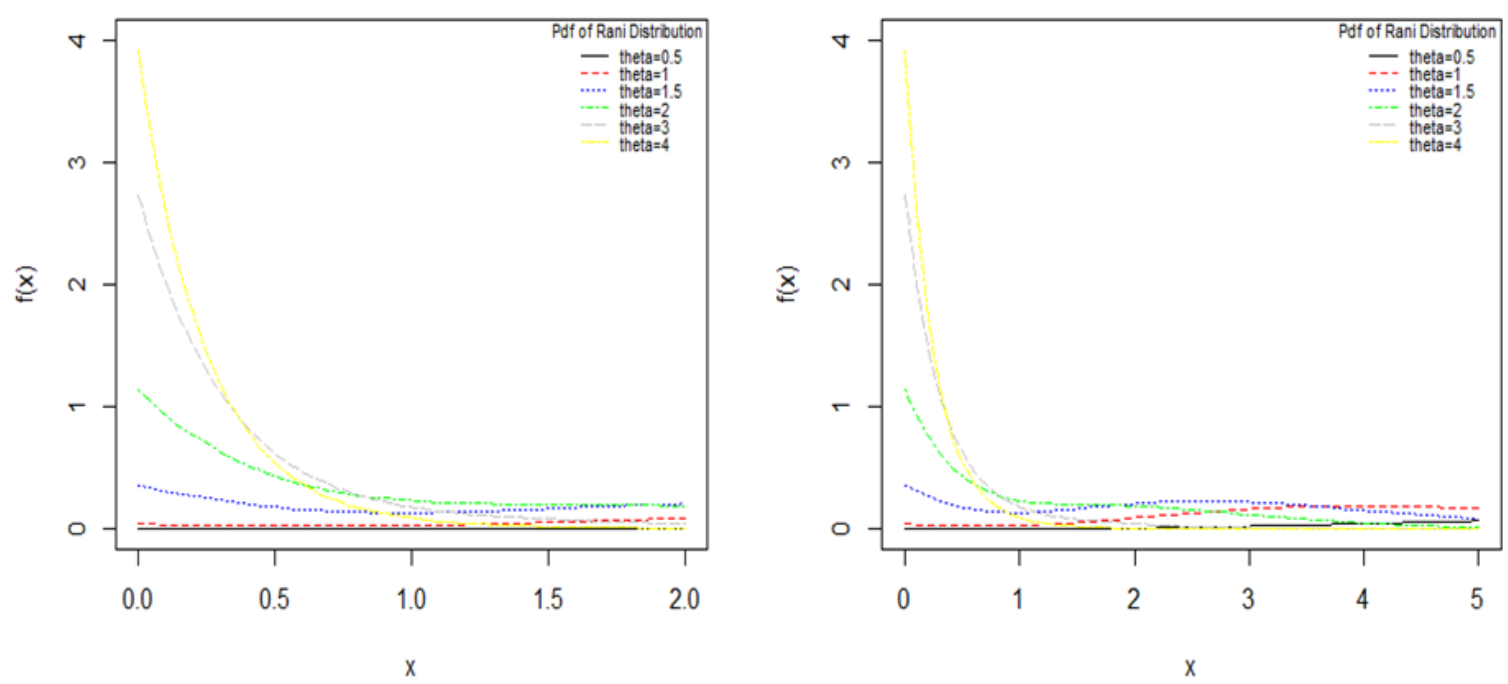

Figure I Graphs of the pdf of Rani distribution for varying values of the parameter $\theta$.
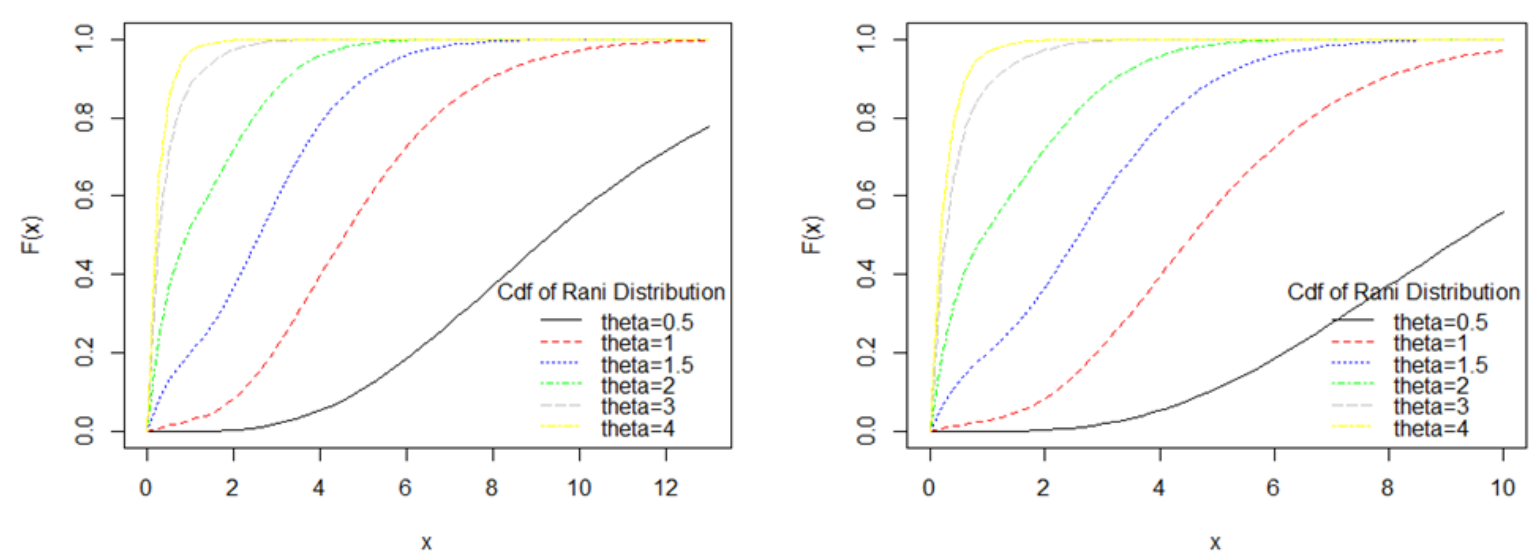

Figure 2 Graphs of the cdf of Rani distribution for varying values of the parameter $\theta$. 


\section{Moments and moments based measures}

The moment generating function of Rani distribution (1.1) can be obtained as

$$
\begin{gathered}
=\frac{\theta^{5}}{\theta^{5}+24}\left[\frac{\theta}{\theta-t}+\frac{24}{(\theta-t)^{5}}\right] \\
=\frac{\theta^{5}}{\theta^{5}+24}\left[\sum_{k=0}^{\infty}\left(\frac{t}{\theta}\right)^{k}+\frac{24}{\theta^{5}} \sum_{k=0}^{\infty}\left(\begin{array}{c}
k+4 \\
k
\end{array}\right)\left(\frac{t}{\theta}\right)^{k}\right] \\
=\sum_{k=0}^{\infty} \frac{\theta^{5}+(k+1)(k+2)(k+3)(k+4)}{\theta^{5}+24}\left(\frac{t}{\theta}\right)^{k}
\end{gathered}
$$

Thus the $r^{\text {th }}$ moment about origin $\mu_{r}^{\prime}$, obtained as the coefficient of $\frac{t^{r}}{r !}$ in $M_{X}(t)$, of Rani distribution can be given by

$$
\mu_{r}^{\prime}=\frac{r !\left[\theta^{5}+(r+1)(r+2)(r+3)(r+4)\right]}{\theta^{r}\left(\theta^{5}+24\right)} ; r=1,2,3, \ldots
$$

Substituting $r=1,2,3$, and 4 , the first four moments about origin of Rani distribution are obtained as

$$
\begin{aligned}
& \mu_{1}^{\prime}=\frac{\theta^{5}+120}{\theta\left(\theta^{5}+24\right)}, \mu_{2}^{\prime}=\frac{2\left(\theta^{5}+360\right)}{\theta^{2}\left(\theta^{5}+24\right)}, \mu_{3}^{\prime}=\frac{6\left(\theta^{5}+840\right)}{\theta^{3}\left(\theta^{5}+24\right)}, \\
& \mu_{4}^{\prime}=\frac{24\left(\theta^{5}+1680\right)}{\theta^{4}\left(\theta^{5}+24\right)}
\end{aligned}
$$

Now using relationship between central moments and moments about origin, the central moments of Rani distribution are obtained as
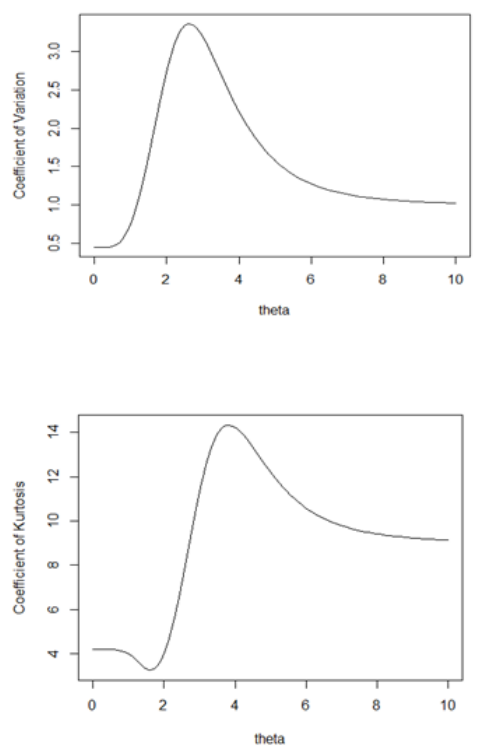

Figure 3 Graphs of coefficient of variation, coefficient of skewness, coefficient of kurtosis and index of dispersion of Rani distribution for varying values of the parameter $\theta$.

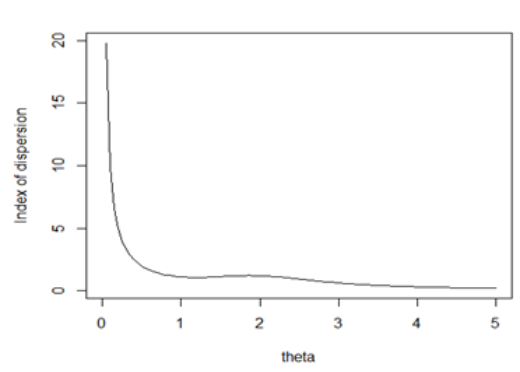

$$
\begin{gathered}
\mu_{3}=\frac{2\left(\theta^{15}+1512 \theta^{10}+1728 \theta^{5}+69120\right)}{\theta^{3}\left(\theta^{5}+24\right)^{3}} \\
\mu_{4}=\frac{9\left(\theta^{20}+2656 \theta^{15}+58752 \theta^{10}+1234944 \theta^{5}+3870720\right)}{\theta^{4}\left(\theta^{5}+24\right)^{4}}
\end{gathered}
$$

The coefficient of variation $(C . V)$, coefficient of skewness $\left(\sqrt{\beta_{1}}\right)$, coefficient of kurtosis $\left(\beta_{2}\right)$ and index of dispersion $(\gamma)$ of Rani distribution are thus obtained as

$$
\begin{gathered}
C . V=\frac{\sigma}{\mu_{1}^{\prime}}=\frac{\sqrt{\theta^{10}+528 \theta^{5}+2880}}{\theta^{5}+120} \\
\beta_{2}=\frac{\mu_{4}}{\mu_{2}^{2}}=\frac{9\left(\theta^{20}+2656 \theta^{15}+58752 \theta^{10}+1234944 \theta^{5}+3870720\right)}{\left(\theta^{10}+528 \theta^{5}+2880\right)^{2}} \\
\gamma=\frac{\sigma^{2}}{\mu_{1}^{\prime}}=\frac{\theta^{10}+528 \theta^{5}+2880}{\theta\left(\theta^{5}+24\right)\left(\theta^{5}+120\right)}
\end{gathered}
$$

The nature of coefficient of variation, coefficient of skewness, coefficient of kurtosis and index of dispersion of Rani distribution have been shown graphically for varying values of parameter in Figure (3). The condition under which Rani distribution is overdispersed, equi-dispersed, and under-dispersed along with condition under which Akash, ${ }^{3}$ Rama, ${ }^{9}$ Akshaya, ${ }^{10}$ Shanker, ${ }^{4}$ Amarendra, ${ }^{7}$ Aradhana, ${ }^{5}$ Sujatha, ${ }^{6}$ Devya, ${ }^{8}$ Lindley ${ }^{1}$ and exponential distributions are over-dispersed, equi-dispersed, and under-dispersed are presented in Table (2).

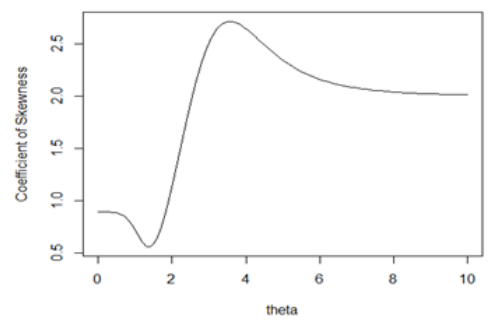


Table 2 Over-dispersion, equi-dispersion and under-dispersion of Rani, Akash, Rama, Akshaya, Shanker, Amarendra, Aradhana, Sujatha, Devya, lindley and exponential distributions for parameter $\theta$

\begin{tabular}{llll}
\hline Distribution & Over-dispersion & Equi-dispersion & Under-dispersion \\
\hline Rani & $\theta<2.449757591$ & $\theta=2.449757591$ & $\theta>2.449757591$ \\
Akash & $\theta<1.515400063$ & $\theta=1.515400063$ & $\theta>1.515400063$ \\
Rama & $\theta<1.950164618$ & $\theta=1.950164618$ & $\theta>1.950164618$ \\
Akshaya & $\theta<1.327527885$ & $\theta=1.327527885$ & $\theta>1.327527885$ \\
Shanker & $\theta<1.171535555$ & $\theta=1.171535555$ & $\theta>1.171535555$ \\
Amarendra & $\theta<1.525763580$ & $\theta=1.525763580$ & $\theta>1.525763580$ \\
Aradhana & $\theta<1.283826505$ & $\theta=1.283826505$ & $\theta>1.283826505$ \\
Sujatha & $\theta<1.364271174$ & $\theta=1.364271174$ & $\theta>1.364271174$ \\
Devya & $\theta<1.451669994$ & $\theta=1.451669994$ & $\theta>1.451669994$ \\
Lindley & $\theta<1.170086487$ & $\theta=1.170086487$ & $\theta>1.170086487$ \\
Exponential & $\theta<1$ & $\theta=1$ & $\theta>1$ \\
\hline
\end{tabular}

\section{Hazard rate function and mean residual life \\ function}

Let $f(x)$ and $F(x)$ be the p.d.f. and c.d.fof a continuous random variable $X$. The hazard rate function (also known as the failure rate function) and the mean residual life function of a continuous random variable $X$ are, respectively, defined as

$$
h(x)=\lim _{\Delta x \rightarrow 0} \frac{P(X<x+\Delta x \mid X>x)}{\Delta x}=\frac{f(x)}{1-F(x)}
$$

and $m(x)=E[X-x \mid X>x]=\frac{1}{1-F(x)} \int_{x}^{\infty}[1-F(t)] d t$

The corresponding hazard rate function, $h(x)$ and the mean residual life function, $m(x)$ of the Rani distribution are obtained as

$$
h(x)=\frac{\theta^{5}\left(\theta+x^{4}\right)}{\theta^{4} x^{4}+4 \theta^{3} x^{3}+12 \theta^{2} x^{2}+24 \theta x+\left(\theta^{5}+24\right)}
$$

and

$$
=\frac{\theta^{4} x^{4}+8 \theta^{3} x^{3}+36 \theta^{2} x^{2}+96 \theta x+\left(\theta^{5}+120\right)}{\theta\left[\theta^{4} x^{4}+4 \theta^{3} x^{3}+12 \theta^{2} x^{2}+24 \theta x+\left(\theta^{5}+24\right)\right]}
$$

It can be easily verified that $h(0)=\frac{\theta^{5}}{\theta^{5}+24}=f(0)$ and $m(0)=\frac{\theta^{5}+120}{\theta\left(\theta^{5}+24\right)}=\mu_{1}^{\prime}$. It is also obvious from the graphs of $h(x)$ and $m(x)$ that the shapes of $h(x)$ is increasing, decreasing and upside bathtub, whereas the shapes of $m(x)$ is decreasing, increasing $(\theta=0.5)$ and downside bathtub. The graphs of the hazard rate function and mean residual life function of Rani distribution are shown in Figure (4).

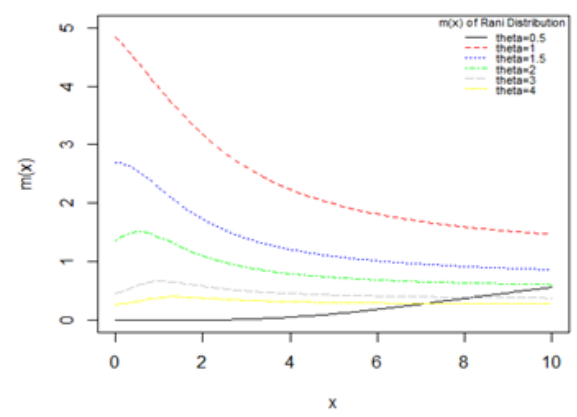

Figure 4 Graphs of $h(x)$ and $m(x)$ of Rani distribution for varying values of the parameter $\theta$. 


\section{Stochastic orderings}

Stochastic ordering of positive continuous random variables is an important tool for judging their comparative behavior. A random variable $X$ is said to be smaller than a random variable $Y$ in the

i. stochastic order $\left(X \leq_{s t} Y\right)$ if $F_{X}(x) \geq F_{Y}(x)$ for all $x$

ii. hazard rate order $\left(X \leq_{h r} Y\right)$ if $h_{X}(x) \geq h_{Y}(x)$ for all $x$

iii. mean residual life order $\left(X \leq_{m r l} Y\right)$ if $m_{X}(x) \leq m_{Y}(x)$ for all iv. likelihood ratio order $\left(X \leq_{l r} Y\right)$ if $\frac{f_{X}(x)}{f_{Y}(x)}$ decreases in $x$.

The following results due to Shaked and Shanthikumar ${ }^{11}$ are well known for establishing stochastic ordering of distributions

$$
\begin{gathered}
X \leq_{l r} Y \Rightarrow X \leq_{h r} Y \Rightarrow X \leq_{m r l} Y \\
\Downarrow \leq_{s t} Y
\end{gathered}
$$

Rani distribution is ordered with respect to the strongest 'likelihood ratio' ordering as shown in the following theorem.

Theorem: Suppose $X \sim$ Rani distributon $\left(\theta_{1}\right)$ and $Y \sim$ Rani distribution $\left(\theta_{2}\right)$. If $\theta_{1}>\theta_{2}$, then $X \leq_{l r} Y$ and hence $X \leq_{h r} Y$, $X \leq_{m r l} Y$ and $X \leq_{s t} Y$.

Proof: We have

$$
\frac{f_{X}(x)}{f_{Y}(x)}=\frac{\theta_{1}^{5}\left(\theta_{2}^{5}+24\right)}{\theta_{2}^{5}\left(\theta_{1}^{5}+24\right)}\left(\frac{\theta_{1}+x^{4}}{\theta_{2}+x^{4}}\right) e^{-\left(\theta_{1}-\theta_{2}\right) x} ; x>0
$$

Now

$$
\ln \frac{f_{X}(x)}{f_{Y}(x)}=\ln \left[\frac{\theta_{1}^{5}\left(\theta_{2}^{5}+24\right)}{\theta_{2}^{5}\left(\theta_{1}^{5}+24\right)}\right]+\ln \left(\frac{\theta_{1}+x^{4}}{\theta_{2}+x^{4}}\right)-\left(\theta_{1}-\theta_{2}\right) x .
$$

This gives $\frac{d}{d x}\left\{\ln \frac{f_{X}(x)}{f_{Y}(x)}\right\}=\frac{-4\left(\theta_{1}-\theta_{2}\right) x^{3}}{\theta_{2}+x^{4}}-\left(\theta_{1}-\theta_{2}\right)$

Thus for $\theta_{1}>\theta_{2}, \frac{d}{d x}\left\{\ln \frac{f_{X}(x)}{f_{Y}(x)}\right\}<0$. This means that $X \leq_{l r} Y$ and hence $X \leq_{h r} Y, X \leq_{m r l} Y$ and $X \leq_{s t} Y$.

\section{Mean deviations}

The amount of scatter in a population is measured to some extent by the totality of deviations usually from mean and median. These are known as the mean deviation about the mean and the mean deviation about the median defined as

$$
\delta_{1}(X)=\int_{0}^{\infty}|x-\mu| f(x) d x \text { and } \delta_{2}(X)=\int_{0}^{\infty}|x-M| f(x) d x
$$
respectively, where $\mu=E(X)$ and $M=\operatorname{Median}(X)$. The measures $\delta_{1}(X)$ and $\delta_{2}(X)$ can be calculated using the simplified relationships

$$
\delta_{1}(X)=\int_{0}^{\mu}(\mu-x) f(x) d x+\int_{\mu}^{\infty}(x-\mu) f(x) d x
$$

$$
\begin{aligned}
& =\mu F(\mu)-\int_{0}^{\mu} x f(x) d x-\mu[1-F(\mu)]+\int_{\mu}^{\infty} x f(x) d x \\
& =2 \mu F(\mu)-2 \mu+2 \int_{\mu}^{\infty} x f(x) d x \\
& =2 \mu F(\mu)-2 \int_{0}^{\mu} x f(x) d x
\end{aligned}
$$

and

$$
\begin{aligned}
& \delta_{2}(X)=\int_{0}^{M}(M-x) f(x) d x+\int_{M}^{\infty}(x-M) f(x) d x \\
& =M F(M)-\int_{0}^{M} x f(x) d x-M[1-F(M)]+\int_{M}^{\infty} x f(x) d x \\
& =-\mu+2 \int_{M}^{\infty} x f(x) d x \\
& =\mu-2 \int_{0}^{M} x f(x) d x
\end{aligned}
$$

Using p.d.f. (1.1) and expression for the mean of Rani distribution (1.1), we get

$\int_{0}^{\mu} x f(x) d x=\mu-\frac{\left\{\theta^{5}\left(\mu^{5}+\theta \mu+1\right)+5 \theta^{4} \mu^{4}+20 \theta^{3} \mu^{3}+60 \theta^{2} \mu^{2}+120(\theta \mu+1)\right\} e^{-\theta \mu}}{\theta\left(\theta^{5}+24\right)}$

$\int_{0}^{M} x f(x) d x=\mu-\frac{\left\{\theta^{5}\left(M^{5}+\theta M+1\right)+5 \theta^{4} M^{4}+20 \theta^{3} M^{3}+60 \theta^{2} M^{2}+120(\theta M+1)\right\} e^{-\theta M}}{\theta\left(\theta^{5}+24\right)}$

Using expressions from (5.1), (5.2), (5.3), and (5.4), the mean deviation about mean, $\delta_{1}(X)$ and the mean deviation about median, $\delta_{2}(X)$ of Rani distribution (1.1) are obtained as

$$
\begin{aligned}
& \delta_{1}(X)=\frac{2\left\{\theta^{4} \mu^{4}+8 \theta^{3} \mu^{3}+36 \theta^{2} \mu^{2}+96 \theta \mu+\left(\theta^{5}+120\right)\right\} e^{-\theta \mu}}{\theta\left(\theta^{5}+24\right)} \\
& \delta_{2}(X)=\frac{2\left\{\begin{array}{l}
\theta^{5}\left(M^{5}+\theta M\right)+5 \theta^{4} M^{4}+20 \theta^{3} M^{3}+60 \theta^{2} M^{2} \\
+120 \theta M+\left(\theta^{5}+120\right)
\end{array}\right\} e^{-\theta M}}{\theta\left(\theta^{5}+24\right)}-\mu
\end{aligned}
$$

\section{Bonferroni and lorenz curves}

The Bonferroni and Lorenz curves ${ }^{12}$ and Bonferroni and Gini indices have applications not only in economics to study income and poverty, but also in other fields like reliability, demography, insurance and medicine. The Bonferroni and Lorenz curves are defined as

$$
B(p)=\frac{1}{p \mu} \int_{0}^{q} x f(x) d x=\frac{1}{p \mu}\left[\int_{0}^{\infty} x f(x) d x-\int_{q}^{\infty} x f(x) d x\right]=\frac{1}{p \mu}\left[\mu-\int_{q}^{\infty} x f(x) d x\right]
$$


and

$$
L(p)=\frac{1}{\mu} \int_{0}^{q} x f(x) d x=\frac{1}{\mu}\left[\int_{0}^{\infty} x f(x) d x-\int_{q}^{\infty} x f(x) d x\right]=\frac{1}{\mu}\left[\mu-\int_{q}^{\infty} x f(x) d x\right]
$$

respectively or equivalently

$$
\begin{array}{r}
B(p)=\frac{1}{p \mu} \int_{0}^{p} F^{-1}(x) d x \\
\text { and } L(p)=\frac{1}{\mu} \int_{0}^{p} F^{-1}(x) d x
\end{array}
$$

respectively, where $\mu=E(X)$ and $q=F^{-1}(p)$.

The Bonferroni and Gini indices are thus defined as

$$
\begin{aligned}
& B=1-\int_{0}^{1} B(p) d p \\
& \text { and } G=1-2 \int_{0}^{1} L(p) d p \text { respectively. }
\end{aligned}
$$

Using p.d.f. of Rani distribution (1.1), we have

$$
\int_{q}^{\infty} x f(x) d x=\frac{\left\{\theta^{5}\left(q^{5}+\theta q\right)+5 \theta^{4} q^{4}+20 \theta^{3} q^{3}+60 \theta^{2} q^{2}+120 \theta q+\left(\theta^{5}+120\right)\right\} e^{-\theta q}}{\theta\left(\theta^{5}+24\right)}
$$

Now using equation (6.7) in (6.1) and (6.2), we have

$$
\begin{aligned}
& B(p)=\frac{1}{p}\left[1-\frac{\left\{\theta^{5}\left(q^{5}+\theta q\right)+5 \theta^{4} q^{4}+20 \theta^{3} q^{3}+60 \theta^{2} q^{2}+120 \theta q+\left(\theta^{5}+120\right)\right\} e^{-\theta q}}{\theta^{5}+120}\right] \\
& \text { and } L(p)=1-\frac{\left\{\theta^{5}\left(q^{5}+\theta q\right)+5 \theta^{4} q^{4}+20 \theta^{3} q^{3}+60 \theta^{2} q^{2}+120 \theta q+\left(\theta^{5}+120\right)\right\} e^{-\theta q}}{\theta^{5}+120}
\end{aligned}
$$

Now using equations (6.8) and (6.9) in (6.5) and (6.6), the Bonferroni and Gini indices are obtained as

$$
\begin{aligned}
& B=1-\frac{\left\{\theta^{5}\left(q^{5}+\theta q\right)+5 \theta^{4} q^{4}+20 \theta^{3} q^{3}+60 \theta^{2} q^{2}+120 \theta q+\left(\theta^{5}+120\right)\right\} e^{-\theta q}}{\theta^{5}+120} \\
& G=\frac{2\left\{\theta^{5}\left(q^{5}+\theta q\right)+5 \theta^{4} q^{4}+20 \theta^{3} q^{3}+60 \theta^{2} q^{2}+120 \theta q+\left(\theta^{5}+120\right)\right\} e^{-\theta q}}{\theta^{5}+120}-1
\end{aligned}
$$

\section{Order statistics and renyi entropy measure}

\section{Distribution of order statistics}

Let $X_{1}, X_{2}, \ldots, X_{n}$ be a random sample of size $n$ from Rani distribution (1.1). Let $X_{(1)}<X_{(2)}<\ldots<X_{(n)}$ denote the corresponding order statistics. The p.d.f. and the c.d.f. of the $k^{\text {th }}$ order statistic, say $Y=X_{(k)}$ are given by

$$
f_{Y}(y)=\frac{n !}{(k-1) !(n-k) !} F^{k-1}(y)\{1-F(y)\}^{n-k} f(y)
$$

$$
=\frac{n !}{(k-1) !(n-k) !} \sum_{l=0}^{n-k}\left(\begin{array}{c}
n-k \\
l
\end{array}\right)(-1)^{l} F^{k+l-1}(y) f(y)
$$

and

$$
\begin{aligned}
& F_{Y}(y)=\sum_{j=k}^{n}\left(\begin{array}{l}
n \\
j
\end{array}\right) F^{j}(y)\{1-F(y)\}^{n-j} \\
& =\sum_{j=k}^{n} \sum_{l=0}^{n-j}\left(\begin{array}{c}
n \\
j
\end{array}\right)\left(\begin{array}{c}
n-j \\
l
\end{array}\right)(-1)^{l} F^{j+l}(y),
\end{aligned}
$$

respectively, for $k=1,2,3, \ldots, n$.

Thus, the p.d.f. and the c.d.f of ${ }^{k}$ th order statistics of Rani distribution are given by

$$
f_{Y}(y)=\frac{n ! \theta^{5}\left(\theta+x^{4}\right) e^{-\theta x}}{\left(\theta^{5}+24\right)(k-1) !(n-k) !} \sum_{l=0}^{n-k}\left(\begin{array}{c}
n-k \\
l
\end{array}\right)(-1)^{l} \times\left[1-\left\{1+\frac{\theta x\left(\theta^{3} x^{3}+4 \theta^{2} x^{2}+12 \theta x+24\right)}{\theta^{5}+24}\right\} e^{-\theta x}\right]^{k+l-1}
$$




$$
\text { and } F_{Y}(y)=\sum_{j=k}^{n} \sum_{l=0}^{n-j}\left(\begin{array}{c}
n \\
j
\end{array}\right)\left(\begin{array}{c}
n-j \\
l
\end{array}\right)(-1)^{l}\left[1-\left\{1+\frac{\theta x\left(\theta^{3} x^{3}+4 \theta^{2} x^{2}+12 \theta x+24\right)}{\theta^{5}+24}\right\} e^{-\theta x}\right]^{j+l}
$$

\section{Renyi entropy measure}

Entropy of a random variable $X$ is a measure of variation of uncertainty. A popular entropy measure is Renyi entropy. ${ }^{13}$ If $X$ is a continuous random variable having probability density function $f($. , then Renyi entropy is defined as

$$
\begin{aligned}
& T_{R}(\gamma)=\frac{1}{1-\gamma} \log \left\{\int f^{\gamma}(x) d x\right\} \\
& \text { where } \gamma>0 \text { and } \gamma \neq 1 .
\end{aligned}
$$

Thus, the Renyi entropy for the Rani distribution (1.1) is obtained as

$$
\begin{aligned}
& T_{R}(\gamma)=\frac{1}{1-\gamma} \log \left[\int_{0}^{\infty} \frac{\theta^{5 \gamma}}{\left(\theta^{5}+24\right)^{\gamma}}\left(\theta+x^{4}\right)^{\gamma} e^{-\theta \gamma x} d x\right] \\
& =\frac{1}{1-\gamma} \log \left[\int_{0}^{\infty} \frac{\theta^{6 \gamma}}{\left(\theta^{5}+24\right)^{\gamma}}\left(1+\frac{x^{4}}{\theta}\right)^{\gamma} e^{-\theta \gamma x} d x\right] \\
& =\frac{1}{1-\gamma} \log \left[\int_{0}^{\infty} \frac{\theta^{6 \gamma}}{\left(\theta^{5}+24\right)^{\gamma}} \sum_{j=0}^{\infty}\left(\begin{array}{l}
\gamma \\
j
\end{array}\right)\left(\frac{x^{4}}{\theta}\right)^{j} e^{-\theta \gamma x} d x\right] \\
& =\frac{1}{1-\gamma} \log \left[\sum_{j=0}^{\infty}\left(\begin{array}{l}
\gamma \\
j
\end{array}\right) \frac{\theta^{6 \gamma-j}}{\left(\theta^{5}+24\right)^{\gamma}} \int_{0}^{\infty} e^{-\theta \gamma x} x^{4 j+1-1} d x\right] \\
& =\frac{1}{1-\gamma} \log \left[\sum_{j=0}^{\infty}\left(\begin{array}{l}
\gamma \\
j
\end{array}\right) \frac{\theta^{6 \gamma-j}}{\left(\theta^{5}+24\right)^{\gamma}} \frac{\Gamma(4 j+1)}{(\theta \gamma)^{4 j+1}}\right] \\
& =\frac{1}{1-\gamma} \log \left[\sum_{j=0}^{\infty}\left(\begin{array}{l}
\gamma \\
j
\end{array}\right) \frac{\theta^{\gamma-1}(1+\theta)^{\gamma-j}}{(\theta+2)^{\gamma}} \frac{\Gamma(4 j+1)}{(\gamma)^{j+1}}\right] \\
& =\frac{1}{1-\gamma} \log \left[\sum_{j=0}^{\infty}\left(\begin{array}{l}
\gamma \\
j
\end{array}\right) \frac{\theta^{6 \gamma-5 j-1}}{\left(\theta^{5}+24\right)^{\gamma}} \frac{\Gamma(4 j+1)}{(\gamma)^{4 j+1}}\right]
\end{aligned}
$$

\section{Stress-strength reliability}

The stress-strength reliability gives the idea about the life of a component which has random strength $X$ that is subjected to a random stress $Y$. When the stress applied to it exceeds the strength, the component fails instantly and the component will function satisfactorily till $X>Y$. Therefore, $R=P(Y<X)$ is a measure of the component reliability and in statistical literature it is known as stress-strength parameter. It has wide applications in almost all areas of knowledge especially in engineering such as structures, deterioration of rocket motors, static fatigue of ceramic components, aging of concrete pressure vessels etc.

Let $X$ and $Y$ be independent strength and stress random variables having Rani distribution (1.1) with parameter $\theta_{1}$ and $\theta_{2}$ respectively. Then the stress-strength reliability $R$ of Rani distribution can be obtained as

$$
\begin{gathered}
R=P(Y<X)=\int_{0}^{\infty} P(Y<X \mid X=x) f_{X}(x) d x \\
=\int_{0}^{\infty} f\left(x ; \theta_{1}\right) F\left(x ; \theta_{2}\right) d x \\
\theta_{1}^{5}\left[\begin{array}{l}
40320 \theta_{2}^{4}+20160 \theta_{2}^{3}\left(\theta_{1}+\theta_{2}\right)+8640 \theta_{2}{ }^{2}\left(\theta_{1}+\theta_{2}\right)^{2}+2880 \theta_{2}\left(\theta_{1}+\theta_{2}\right)^{3} \\
+24\left(\theta_{2}^{5}+\theta_{1} \theta_{2}^{4}+24\right)\left(\theta_{1}+\theta_{2}\right)^{4}+24 \theta_{1} \theta_{2}^{3}\left(\theta_{1}+\theta_{2}\right)^{5}+24 \theta_{1} \theta_{2}{ }^{2}\left(\theta_{1}+\theta_{2}\right)^{6} \\
+24 \theta_{1} \theta_{2}\left(\theta_{1}+\theta_{2}\right)^{7}+\theta_{1}\left(\theta_{2}{ }^{5}+24\right)\left(\theta_{1}+\theta_{2}\right)^{8}
\end{array}\right] \\
\left(\theta_{1}^{5}+24\right)\left(\theta_{2}^{5}+24\right)\left(\theta_{1}+\theta_{2}\right)^{9}
\end{gathered}
$$

\section{Estimation of parameter}

\section{Maximum likelihood estimate (MLE)}

Let $\left(x_{1}, x_{2}, x_{3}, \ldots, x_{n}\right)$ be a random sample from Rani distribution (1.1). The likelihood function, $L$ of (1.1) is given by

$$
L=\left(\frac{\theta^{5}}{\theta^{5}+24}\right)^{n} \prod_{i=1}^{n}\left(\theta+x_{i}^{4}\right) e^{-n \theta \bar{x}}
$$

The natural log likelihood function is thus obtained as

$$
\begin{gathered}
\ln L=n \ln \left(\frac{\theta^{5}}{\theta^{5}+24}\right)+\sum_{i=1}^{n} \ln \left(\theta+x_{i}^{4}\right)-n \theta \bar{x} . \\
\text { Now } \frac{d \ln L}{d \theta}=\frac{5 n}{\theta}-\frac{5 n \theta^{4}}{\theta^{5}+24}+\sum_{i=1}^{n} \frac{1}{\theta+x_{i}^{4}}-n \bar{x}, \text { where } \bar{x} \text { is the }
\end{gathered}
$$
sample mean.

The MLE $\hat{\theta}$ of $\theta$ is the solution of the equation $\frac{d \ln L}{d \theta}=0$ and thus it is the solution of the following nonlinear equation

$$
\frac{5 n}{\theta}-\frac{5 n \theta^{4}}{\theta^{5}+24}+\sum_{i=1}^{n} \frac{1}{\theta+x_{i}^{4}}-n \bar{x}=0
$$

\section{Method of moment estimate (MOME)}

Equating the population mean of Rani distribution (1.1) to the corresponding sample mean, MOME $\tilde{\theta}$, of $\theta$ is the solution of the following six degree polynomial equation 


$$
\bar{x} \theta^{6}-\theta^{5}+24 \theta \bar{x}-120=0 .
$$

\section{A simulation study}

In this section, a simulation study has been carried out to know the efficiency of the maximum likelihood estimate(MLE) of Rani distribution. The simulation study is based on Acceptance/Rejection method.

\section{Acceptance/Rejection algorithm:}

To simulate from the density $f_{X}$, it is assumed that we have envelope density $h$ from which it can simulate and that we have some $k<\infty$ such that $\operatorname{Sup}_{x} \frac{f_{X}(x)}{h(x)} \leq k$.

Step 1. Simulate $X$ from $h$

Step 2. Generate $Y \sim U(0, k h(x))$, where $k=\frac{\theta^{5}}{\theta^{5}+24}$

Step 3. If $Y<f_{X}(x)$, then return $X$, otherwise go to step 1

The simulation study is based on generating $N=10,000$ samples of size $n=50,100,150,200$ for $\theta=0.5,1,1.5$ and 2 using above algorithm. Then we calculate the following measures

(i) Average bias of the simulated estimate

$$
\text { Average bias }=\frac{1}{N} \sum_{i=1}^{N}\left(\hat{\theta}_{i}-\theta\right) \text {, where } \hat{\theta}_{i} \text { is the ML estimate }
$$

(ii) Average mean square error (MSE)

$$
\text { Average mean square error }=\frac{1}{N} \sum_{i=1}^{N}\left(\hat{\theta}_{i}-\theta\right)^{2} .
$$

The average bias and average mean square error (MSE) for each of the ML estimate has been calculated and shown in Table (3), where MSE has been shown in bracket.

Table 3 Average bias and average mean square error of the simulated estimate

\begin{tabular}{lllll}
\hline$n$ & $\theta=0.5$ & $\theta=1$ & $\theta=1.5$ & $\theta=2$ \\
\hline 50 & 0.05034 & 0.026212 & 0.010078 & -0.00079 \\
& -0.12673 & -0.03435 & -0.01008 & \\
& 0.025405 & 0.132465 & 0.005188 & -0.00033 \\
100 & & & & \\
& -0.06454 & -0.01755 & -0.00269 & \\
150 & 0.017098 & 0.008916 & 0.003523 & -0.0002 \\
& -0.04385 & -0.01193 & -0.00186 & \\
& 0.012992 & 0.006755 & 0.002713 & -0.00012 \\
& & & & \\
& -0.03376 & -0.00913 & -0.00147 & \\
\hline
\end{tabular}

The graphical presentation of MSE for different values of parameter is shown in Figure 5.

\section{Goodness of fit}

In this section, the goodness of fit of Rani distribution has been discussed with a real lifetime data set from engineering and the fit has been compared with one parameter lifetime distributions namely Akash, ${ }^{3}$ Shanker, ${ }^{4}$ Amarendra, ${ }^{7}$ Aradhana,${ }^{5}$ Sujatha,,${ }^{6}$ Devya,${ }^{8}$ Lindley ${ }^{1}$ and exponential. The data set is the strength data of glass of the aircraft window reported by Fuller, et al., ${ }^{14}$ and are given as 18.83 , $20.80,21.657,23.03,23.23,24.05,24.321,25.50,25.52,25.80$, $26.69,26.77,26.78,27.05,27.67,29.90,31.11,33.20,33.73,33.76$, $33.89,34.76,35.75,35.91,36.98,37.08,37.09,39.58,44.045,45.29$, 45.381. In order to compare lifetime distributions, values of $-2 \ln L$, AIC (Akaike Information Criterion) and K-S Statistic ( KolmogorovSmirnov Statistic) for the above data set have been computed and presented in Table (4).

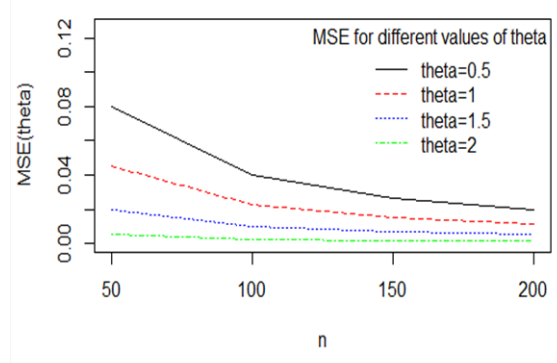

Figure 5 Graphs of MSE for different values of $\theta$ and $n$

The formulae for computing AIC and K-S Statistic are as follows:

$$
A I C=-2 \ln L+2 k, \quad K-S=\operatorname{Sup}_{x}\left|F_{n}(x)-F_{0}(x)\right|, \quad \text { where }
$$

$k=$ the number of parameters, $n=$ the sample size and $F_{n}(x)$ is the empirical distribution function. The best distribution is the distribution which corresponds to lower values of $-2 \ln L$, AIC, and $\mathrm{K}-\mathrm{S}$ statistic and higher p-value. The $\operatorname{MLE}(\hat{\theta})$ with the standard error, S.E $(\hat{\theta})$ of $\theta,-2 \ln L$, AIC, K-S Statistic and p-value of the fitted distributions are presented in the Table (4). It can be easily observed from above Table (3) that Rani distribution gives better fit than the fit given by Akash, ${ }^{3}$ Rama,,${ }^{9}$ Akshaya,${ }^{10}$ Shanker, ${ }^{4}$ Amarendra, ${ }^{7}$ Aradhana, ${ }^{5}$ Sujatha,${ }^{6}$ Devya,${ }^{8}$ Lindley ${ }^{1}$ and exponential distributions and hence it can be considered as an important lifetime distribution for modeling lifetime data over these distributions.

\section{Concluding remarks}

A one parameter lifetime distribution named, "Rani distribution" has been proposed. Its statistical properties including shapes, moments, skewness, kurtosis, index of dispersion, hazard rate function, mean residual life function, stochastic ordering, mean deviations, Bonferroni and Lorenz curves and stress-strength reliability have been discussed. The condition under which Rani distribution is over-dispersed, equi-dispersed, and under-dispersed are presented along other one parameter lifetime distributions. Maximum likelihood estimation and 
method of moments have been discussed for estimating its parameter. A simulation study has been presented. Finally, the goodness of fit test using K-S Statistic (Kolmogorov-Smirnov Statistic) and p-value for a real lifetime data has been presented and the fit has been compared with some one parameter lifetime distributions.
NOTE: The paper is named "Rani distribution" in the name of my lovely niece Rani Kumari, second daughter of my respected eldest brother Professor Shambhu Sharma, Department of Mathematics, Dayalbagh Educational Institute, Dayalbagh, Agra, India.

Table 4 MLE's, S.E - 2ln L,AIC and K-S statistics of the fitted distributions of the given data set

\begin{tabular}{|c|c|c|c|c|c|c|}
\hline Distributions & MLE & S.E & $-2 \ln L$ & AIC & K-S & p-value \\
\hline Rani & 0.162278 & 0.013034 & 227.25 & 229.25 & 0.223 & 0.0775 \\
\hline Akash & 0.097065 & 0.010048 & 240.68 & 242.68 & 0.298 & 0.0059 \\
\hline Rama & 0.129782 & 0.011651 & 232.79 & 234.79 & 0.253 & 0.0301 \\
\hline Akshaya & 0.125745 & 0.011292 & 234.44 & 236.44 & 0.263 & 0.0223 \\
\hline Shanker & 0.647164 & 0.0082 & 252.35 & 254.35 & 0.358 & 0.0004 \\
\hline Amarendra & 0.128294 & 0.012413 & 233.41 & 235.41 & 0.257 & 0.0269 \\
\hline Aradhana & 0.094319 & 0.00978 & 242.22 & 244.22 & 0.306 & 0.0044 \\
\hline Sujatha & 0.095613 & 0.009904 & 241.5 & 243.5 & 0.303 & 0.0051 \\
\hline Devya & 0.160873 & 0.012916 & 227.68 & 229.68 & 0.422 & 0 \\
\hline Lindley & 0.062992 & 0.008001 & 253.98 & 255.98 & 0.365 & 0.0003 \\
\hline Exponential & 0.032449 & 0.005822 & 274.52 & 276.53 & 0.458 & 0 \\
\hline
\end{tabular}

\section{Acknowledgements}

None.

\section{Conflict of interest}

None.

\section{References}

1. Lindley DV. Fiducial distributions and Bayes' theorem. Journal of the Royal Statistical Society Series. 1958;B20:102-107.

5. Shanker R, Hagos F. On modeling of Lifetimes data using exponential and Lindley distributions. Biometrics \& Biostatistics International Journal. 2015;2(5):1-9.

6. Shanker R. Akash distribution and its Applications. International Journal of Probability and Statistics. 2015 a;4(3):65-75.

7. Shanker R. Shanker distribution and its Applications. International Journal of Statistics and Applications. 2015b;5(6):338-348.

8. Shanker R. Aradhana distribution and its Applications. International Journal of Statistics and Applications. 2016 c;6(1):23-34.

9. Shanker R. Sujatha distribution and its Applications. Statistics in Transition-new series. 2016 d;17(3):1-20.
10. Shanker R. Amarendra distribution and its Applications. American Journal of Mathematics and Statistics. 2016 a;6(1):44-56.

11. Shanker R. Devya distribution and its Applications. International Journal of Statistics and Applications. 2016 b;6(4):189-202.

12. Shanker R. Rama distribution and its Application. International Journal of Statistics and Applications. 2017 a;7(1):26-35.

13. Shanker R. Akshaya distribution and its Application. American Journal of Mathematics and Statistics. 2017 b;7(2):51-59.

14. Shaked M and Shanthikumar JG. Stochastic Orders and Their Applications Academic Press, New York, USA. 1994.

15. Bonferroni CE. Elementi di Statistca generale Seeber Firenze.

16. Renyi A. On measures of entropy and information in proceedings of the $4^{\text {th }}$ berkeley symposium on Mathematical Statistics and Probability Berkeley. Univ of Cali Press. 1961;1:547-561.

17. Fuller E J, Frieman S, Quinn J, et al. Fracture mechanics approach to the design of glass aircraft windows: A case study. SPIE Proc. 1994;2286:419-430. 